\title{
COST ANALYSIS OF TAXANE-BASED AND CISPLATIN-BASED CHEMOTHERAPY REGIMENS FOR EPITHELIAL OVARIAN CANCER IN DHARMAIS NATIONAL CANCER HOSPITAL
}

\author{
BAMBANG DWIPOYONO ${ }^{1 *}$, SEPTYANA CHOIRUNISA ${ }^{2}$, MARDIATI NADJIB ${ }^{3}$, AMAL C SJAAF ${ }^{3}$
}

${ }^{1}$ Department of Obstetrics and Gynecology, Dharmais National Cancer Hospital, Jakarta, Indonesia. ${ }^{2}$ Department of Pharmacy, Faculty of Public Health, Universitas Indonesia, Depok, Indonesia. ${ }^{3}$ Department of Health Policy and Administration, Faculty of Public Health, Universitas Indonesia, Depok, Indonesia. Email: bdwipoyono@gmail.com

Received: 21 April 2017, Revised and Accepted: 18 August 2017

\section{ABSTRACT}

Objective: This exploratory study aimed to evaluate and compare the treatment costs of taxane-based versus cisplatin-based chemotherapy.

Methods: This study used data from the medical and financial records of ovarian cancer patients who were admitted to Dharmais National Cancer Hospital (RSKD) between 2008 and 2012 and subsequently underwent surgery and were treated with chemotherapy. Data were analyzed using descriptive analysis, and a Kaplan-Meier graph was plotted to compare the survival of the patients in the taxane-based and cisplatin-based chemotherapy groups.

Results: Of 41 patients, treatment costs were available for nine patients who had undergone taxane-based chemotherapy and for 31 patients who had undergone cisplatin-based chemotherapy. In general, surgical procedures accounted for the highest proportion of the treatment costs, followed by chemotherapy. Taxane-based chemotherapy (six cycles) was 4 times more expensive than cisplatin-based therapy. The pre- and post-chemotherapy costs of care among those treated with the taxane-based regimen were 3-4 times more expensive than those of the patients who received cisplatinbased treatment. The disease-free recurrence duration of the patients treated with taxane was longer (median=18 months) than that of the patients treated with cisplatin (median $=5$ months).

Conclusions: Taxane-based therapy increased the disease-free recurrence duration of the patients, with disease-free recurrence 3 times longer than that of the patients treated with the cisplatin-based regimen. However, the treatment costs of the taxane-based regimen were 4 times higher than those of the cisplatin-based treatment.

Keywords: Ovarian cancer, Cost analysis, Taxane chemotherapy, Cisplatin chemotherapy.

(C) 2017 The Authors. Published by Innovare Academic Sciences Pvt Ltd. This is an open access article under the CC BY license (http://creativecommons. org/licenses/by/4. 0/) DOI: http://dx.doi.org/10.22159/ijap.2017.v9s1.82_89

\section{INTRODUCTION}

Epithelial ovarian cancer remains the most lethal gynecologic malignancy in the world [1-3]. The global incidence of ovarian cancer is approximately 190,000 cases/year and varies depending on the country, with $15-20$ cases $/ 100,000$ in developed countries (i.e., U.S. and U.K.), 8-10 cases/100,000 in Southern Europe, and $<7$ cases $/ 100,000$ in China and Japan [4]. The number of cases in developing countries, such as Indonesia, is undetermined due to a lack of registration data [5]. However, ovarian cancer is estimated to be the second most common gynecological cancer after cervical cancer [6].

The 5-year survival rate of ovarian cancer patients in Indonesia is estimated to be between $34.5 \%$ and $54.8 \%$, according to data from Cipto Mangunkusumo National Referral Hospital [6] and Dharmais National Cancer Hospital [7]. Although most ovarian cancer patients have a positive response for surgery and chemotherapy [8], recurrence occurs in $60-70 \%$ of cases within 3 years [9]. The median disease-free survival of patients with Stage III disease in Western European countries ranges between 15 and 17 months, whereas the median disease-free survival of patients with Stage IV disease in these countries is 8-9 months [10].

Surgery and chemotherapy remain the standard treatments for Stage IC ovarian cancer and higher [11]. Chemotherapy regimens have led to improvements in patient outcomes [11]. As reported by Cristea et al. [11], monotherapy, which was developed in the 1960s, resulted in a response in $13-30 \%$ of patients. Monotherapy was followed in the 1980 s by combination therapy with cisplatin and cyclophosphamide [11]. The latter was associated with a positive response in $60 \%$ of patients [11].
Taxane, introduced in the 1990s, showed the best response (70\%) [11]. Before 2006, a combination of cisplatin and cyclophosphamide was used for ovarian cancer in Dharmais Cancer Hospital. After 2006, the treatment protocol for ovarian cancer at the hospital changed to cisplatin- and taxane-based regimens [12]. Although these chemotherapy regimens are commonly used for ovarian cancer, no cost evaluations of these treatments have been conducted.

\section{METHODS}

This was an explorative retrospective cohort study aimed at analyzing the unit cost components of inpatient care. The study population consisted of patients with epithelial ovarian cancer who were admitted to Dharmais Cancer Hospital between 2008 and 2012, underwent a complete treatment program based on the hospital's protocol, and had a minimum follow-up of 2 years, in addition to a complete medical and billing record. Patients with incomplete treatment were excluded due to difficulties in assessing outcomes. The exposed group consisted of patients who received taxane-based chemotherapy, and the nonexposed group consisted of patients who received cisplatin-based chemotherapy.

Forty-one patients fulfilled the inclusion and exclusion criteria. Three patients with complete cost records who underwent surgery and cisplatin-based chemotherapy and nine patients who underwent surgery and taxane-based chemotherapy were selected. As this was an explorative study, data on only about $30 \%$ of cases in each chemotherapy group were analyzed due to their feasibility, not due to their representativeness. 
To obtain surgery costs, data were collected on 13 patients who underwent surgery only. This was done due to the difficulty in obtaining surgery only costs on the patients who underwent both surgery and chemotherapy. However, the characteristics of the patients who underwent surgery only were similar to those who underwent both surgery and chemotherapy, as the surgery procedure in both groups adhered to standard protocols adopted at Dharmais Cancer Hospital.

Patients' medical data were obtained from their medical records. The data included age, education level, insurance, parity, cancer stage, complete/incomplete surgery, hemoglobin, albumin, Ca125 presurgery levels, and cancer recurrence. Surgery-related costs included operating theater, intensive care unit/high care unit, ward, drug, examination of surgical specimen, laboratory test, and blood transfusion costs. Chemotherapy-related costs included pre- and post-chemotherapy expenses. These included the chemotherapy regimen, drugs for treating side effects and complications, ward fees, doctors' fees, and any supporting material costs.

\section{Statistical analysis}

A descriptive analysis of the cost-related data was conducted. A Kaplan-Meier graph was plotted to compare the survival of the patients in the taxane-based and cisplatin-based chemotherapy groups.

\section{RESULTS}

As shown in Table 1, the patients who received cisplatin-based chemotherapy were younger (median: 42 years) than those who received taxane-based chemotherapy (median: 51 years). The hemoglobin level of more than half of the patients in the taxane group (81.3\%) was above $10 \mathrm{~g} / \mathrm{dL}$, whereas it exceeded this level in only $18.8 \%$ of the patients in the cisplatin group.

In general, surgical procedures accounted for the highest treatment costs among all the cost components (pre-chemotherapy, chemotherapy, and post-chemotherapy). In the surgical subcomponent, the costs for operating rooms, medical personnel, and medicine accounted for the highest costs. The total cost of the surgery was approximately IDR 80-97 million. Operating room costs accounted for the highest proportion $(23.4 \%)$ of the total costs, followed by operational costs for doctors (20\%, approximately IDR 22.8 million), medicine(15$20 \%$,approximately IDR 12-20 million), intensive/high care unit or ward $(7.5 \%$, approximately IDR 5.8 million), and finally, medical consumable materials (3\%).

The second highest cost incurred after surgery was the cost of chemotherapy. The highest subcomponent cost of chemotherapy was the medicine/regimen. The cost of each cycle of taxane-based chemotherapy was approximately IDR 7.1 million $(53 \%$ from 13.4 million). The cost of each cycle of cisplatin-based chemotherapy was IDR 1.5 million (50\% from 3.1 million). Six cycles of taxane-based chemotherapy were approximately 4 times more expensive than cisplatin-based chemotherapy, with a cost of IDR 80 million for the former compared to IDR 18 million for the latter. The pre- and postchemotherapy costs of the care of those treated with the taxane-based

Table 1: Characteristic of the ovarian cancer patients

\begin{tabular}{|c|c|c|c|c|}
\hline \multirow[t]{3}{*}{ Variables } & \multicolumn{2}{|c|}{ Type of chemotherapy } & \multirow[t]{3}{*}{ Total } & \multirow[t]{3}{*}{$\mathbf{p}$} \\
\hline & \multicolumn{2}{|l|}{ n (\%) } & & \\
\hline & Cisplatin $(n=9)$ & Taxane $(n=31)$ & & \\
\hline Age (years), mean & $45.9 \pm 4.5$ & $50.3 \pm 1.0$ & & \\
\hline Age (years), median & 42 & 51 & & \\
\hline Min-max & $35-77$ & $40-62$ & & \\
\hline \multicolumn{5}{|l|}{ Age group (years) } \\
\hline$\leq 50$ & $7(33.3)$ & $14(66.7)$ & 21 & 0.083 \\
\hline $51-60$ & $1(5.9)$ & $16(94.1)$ & 17 & \\
\hline$>60$ & $1(50)$ & $1(50)$ & 2 & \\
\hline \multicolumn{5}{|l|}{ Education level } \\
\hline Secondary & 7 (26.9) & $19(73.1)$ & 26 & 0.391 \\
\hline Primary & $1(9.1)$ & $10(90.9)$ & 11 & \\
\hline \multicolumn{5}{|l|}{ Source of fund } \\
\hline Insurance & $4(25)$ & $12(75)$ & 16 & \\
\hline No Insurance & $4(19)$ & $17(81)$ & 21 & \\
\hline \multicolumn{5}{|l|}{ Parity } \\
\hline $0-1$ & $4(30.8)$ & $9(69.2)$ & 13 & 0.613 \\
\hline \multirow{2}{*}{\multicolumn{5}{|c|}{ Stage }} \\
\hline & & & & \\
\hline II & $2(25)$ & $6(75)$ & 8 & 0.607 \\
\hline II and IV & $7(22.6)$ & $24(77.4)$ & 31 & \\
\hline \multicolumn{5}{|l|}{ Surgery } \\
\hline Complete & $5(38.5)$ & $8(61.5)$ & 13 & 0.348 \\
\hline Incomplete & $1(16.7)$ & $5(83.3)$ & 6 & \\
\hline \multicolumn{5}{|c|}{ Presurgery hemoglobin level (g/dL) } \\
\hline$<10$ & $3(42.9)$ & $4(57.1)$ & 7 & 0.187 \\
\hline$\geq 10$ & $6(18.8)$ & $26(81.3)$ & 32 & \\
\hline \multicolumn{5}{|c|}{ Presurgery albumin level (mg/dL) } \\
\hline$\leq 3.5^{\circ}$ & $4(21.1)$ & $15(78.9)$ & 19 & 0.506 \\
\hline$>3.5$ & $4(26.7)$ & $11(73.3)$ & 15 & \\
\hline \multicolumn{5}{|c|}{ Presurgery Ca125 level } \\
\hline$\leq 35$ & $0(0)$ & $4(100)$ & 4 & 0.365 \\
\hline $35.1-200$ & $2(40)$ & $3(60)$ & 5 & \\
\hline$>200$ & $4(23.5)$ & $13(76.5)$ & 17 & \\
\hline \multicolumn{5}{|l|}{ Recurrence } \\
\hline Yes & $3(20)$ & $12(80)$ & 15 & 0.519 \\
\hline No & $3(37.5)$ & $5(62.5)$ & 8 & \\
\hline Lost to follow up & $3(20)$ & $12(80)$ & 15 & \\
\hline
\end{tabular}


Table 2: Treatment costs of ovarian cancer patients based on the type of chemotherapy

\begin{tabular}{|c|c|c|c|c|c|}
\hline Variables & $\mathbf{n}$ & Cisplatin based & $\%$ Cost & Taxane based & $\%$ Cost \\
\hline Surgery & 13 & Rp. 81.133.836 & 78 & Rp. 81.133.836 & 45.7 \\
\hline Chemotherapy/6 cycles & 12 & Rp. 18.822 .666 & 18 & Rp. 80.739 .390 & 45.4 \\
\hline Pre-chemotherapy/6 cycles & 1 & Rp. 1.880 .964 & 2 & Rp. 6.130 .410 & 3.5 \\
\hline Post-chemotherapy/ 6 cycles & 1 & Rp. 2.436 .216 & 2 & Rp. 9.660 .432 & 5.4 \\
\hline Total costs & & Rp. 104.273 .682 & 100 & Rp. 177.664 .068 & 100.0 \\
\hline
\end{tabular}

regimen were 3-4 times higher than those treated with the cisplatinbased regimen. Treatment costs of ovarian cancer patients based on the type of chemotherapy are described in Table 2.

Based on the cumulative survival probability, the patients treated with taxane were more likely to have a higher survival probability (50\%) and longer disease-free duration (median=18 months) than the patients who were treated with cisplatin (survival probability $=0 \%$; median $=5$ months). Overall, the results showed that the disease-free duration of the patients who received the taxane-based chemotherapy was 3 times longer than that of the patients who were treated with the cisplatin-based regimen. Cumulative survival probability of ovarian cancer patients based on the type of chemotherapy is described in Fig. 1. Median disease-free survival based on a 2-year follow-up is shown in Table 3.

\section{DISCUSSION}

\section{Costs of surgery}

The surgery-related cost of ovarian cancer in Indonesia was approximately IDR 81 million. This cost was lower than the reported cost in the U.S., which was between $\$ 200$ and $\$ 300$ million U.S. dollars in the years up to and including 2004 [13].

\section{Chemotherapy cost}

The mean cost of cisplatin-based chemotherapy in Indonesia was IDR 18 million or roughly one-fourth cheaper than that of taxanebased chemotherapy, which cost IDR 80 million for six cycles. The components that contributed to the direct cost of chemotherapy were medication, pre- and post-chemotherapy medication, and adverse effects, which incurred additional personal or examination costs [13]. The incremental cost for taxane-based therapy per life-year saved was IDR 95 million or equal to $\$ 7,056$. Its cost effectiveness was better than that reported in Spain $(\$ 6,395)$, France $(\$ 6,642)$, and the U.K. $(\$ 6,403)$ but poorer than that reported in the Netherlands $(\$ 7,796)$, Germany $(\$ 9,362)$, and Italy $(\$ 11,420)[14,15]$.

The approximate pre- and post-chemotherapy costs of the taxane-based therapy, including adverse effects of the treatment, were 6.1 and IDR 9.6 million, respectively. For the cisplatin-based regimen, these costs were IDR 1.8 and 2.4 million, respectively. The pre- and post-chemotherapy costs of the taxane-based chemotherapy were 3-4 times higher than those incurred with cisplatin-based chemotherapy. According to the literature, adverse effects of taxane-based chemotherapy include neurotoxicity and hematological disturbances [16].

Although a standard protocol is in place at Dharmais Cancer Hospital for ovarian cancer treatment, the doctor's preference and the procurement process, including the price of medications, discounts, and availability, lead to variations in the use of ovarian cancer medications. These variations result in differences in treatment costs. The procurement process for medical materials and equipment at Dharmais Cancer Hospital also affect total treatment costs. In this study, the cost included the cost of medical materials and equipment used in surgery procedure but excluded the cost of medical materials and equipment in the chemotherapy procedure because of the insignificant nature of these costs as compared to the total cost of medications and medical services.

Reviews on the Health Technology Assessment and clinical pathway implementation are expected to improve the efficiency of the service
Table 3: Median disease-free survival based on a 2-year follow-up

\begin{tabular}{lll}
\hline $\begin{array}{l}\text { Diseases- } \\
\text { free survival }\end{array}$ & Time (months) & \\
\cline { 2 - 3 } & Cisplatin based (n=6) & Taxane based $(\mathbf{n = 2 9 )}$ \\
\hline Mean & $13.7 \pm 4.23(\mathrm{SE})$ & $16.6 \pm 1.6(\mathrm{SE})$ \\
Median & $5(\mathrm{SE}$, none) & $18 \pm 4.8(\mathrm{SE})$ \\
\hline
\end{tabular}

SE: Standard error

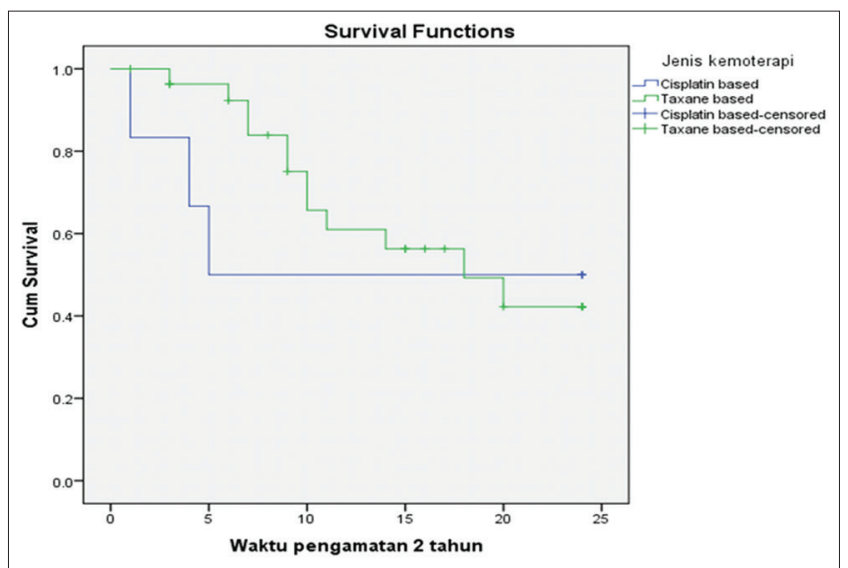

Fig. 1: Cumulative survival probability of ovarian cancer patients based on the type of chemotherapy

by lowering service costs and lengths of hospital stays, in addition to improving the quality of the service [17].

\section{Recurrence}

Based on a 2-year follow-up, the cumulative survival probability of the patients who received the cisplatin-based and taxane-based therapies was $\pm 50 \%$ and $\pm 40 \%$, respectively, with a lost to follow-up rate of $34.3 \%$. The mean and median period of recurrence in the taxane-based chemotherapy group was 16.6 months and 18 months, respectively. In the cisplatin-based chemotherapy group, the mean and median period of recurrence was 13.7 months and 5 months, respectively. The results for medial survival were similar to those found in the GOG 111-Dutch/ Danish-AGO-GOG 158 study, which reported that the median survival of patients who received taxane-based treatment was 16-19.4 months [18]. However, the median survival of patients who received cisplatin-based treatment in the Dutch/Danish study was different (11.5-13 months).

\section{CONCLUSION}

The total cost of taxane-based chemotherapy was 4-5 times higher than the cost of cisplatin-based therapy. The mean and median period of recurrence were 13.7 months and 5 months, respectively, in the cisplatinbased group, and it was 16.6 months and 18 months, respectively, in the taxane-based group. The probability of non-recurrence in the patients who received cisplatin-based chemotherapy was $50 \%$, whereas it was $42 \%$ in the taxane-based group. This study found that a taxane-based regimen used as adjuvant chemotherapy was 3 times more protective against the recurrence of ovarian cancer as compared to cisplatin-based chemotherapy. 


\section{REFERENCES}

1. Kurman RJ, Shih IeM. the origin and pathogenesis of epithelial ovarian cancer: A proposed unifying theory. Am J Surg Pathol 2010;34(3):433-43.

2. Jelovac D, Armstrong DK. Recent progress in the diagnosis and treatment of ovarian cancer. CA Cancer J Clin 2011;61(3):183-203.

3. Hollis RL, Gourley C. Genetic and molecular changes in ovarian cancer. Cancer Biol Med 2016;13(2):236-47.

4. Parkin DM, Pisani P, Ferlay J. Global Cancer Statistics. CA Cancer J Clin 1999;49(1):33-64

5. Ferlay J, Bray F, Pisani P, Parkin DM. GLOBOCAN 2002: Cancer incidence, mortality and prevalence worldwide. Lyon: IARC Press;2004.

6. Aziz MF. Gynecological cancer in Indonesia. J Gynecol Oncol 2009;20(1):8-10.

7. Ubiet JF. Angka kesintasan kanker ovarium di RS Kanker Dharmais. Thesis. Depok: Universitas Indonesia;2009. [In Indonesia].

8. Ozols RF, Bundy BN, Greer BE, Fowler JM, Clarke-Pearson D, Burger RA, et al. Phase III trial of carboplatin and paclitaxel compared with cisplatin and paclitaxel in patients with optimally resected stage III ovarian cancer. J Clin Oncol 2003;21(7):3194-200.

9. Prat A, parera M, Del Campo JM. Prognostic role of CA-125 nadir in stage IV epithelial ovarian cancer. J Clin Oncol 2008;26(10):1771-2.
10. Inciura A, Simavicius A, Juozaityte E, Kurtinaitis J, Nadisauskiene R, Svedas E, et al. Comparison of adjuvant and neoadjuvant chemotherapy in the management of advanced ovarian cancer: A retrospective study of 574 patients. BMC cancer 2006;6(1):153.

11. Cristea M, Han E, Salmon L, Morgan RJ. Review: Practical considerations in ovarian cancer chemotherapy. Ther Adv Med Oncol 2010;2(3):175-87.

12. Rumah Sakit Kanker Dharmais. Gynaecological cancer protocol. Jakarta: RSKD; 2001. [In Indonesia].

13. Reeder CE, Gordon D. Managing oncology costs. Am J Manag Care 2006;12(1 Suppl):S3-16.

14. Expósito J, Hernández J, Feijóo AF, Nieto T, Briones E. New chemotherapy treatments in advanced cancer patients an easily applicable evaluation of clinical efficacy and cost-effectiveness. Acta Oncol 2003;42(8):895-902

15. Sfakianos GP, Havrilesky LJ. A review of cost-effectiveness studies in ovarian cancer. Cancer Control 2011;18(1):59-64

16. Cella D, Peterman A, Hudgens S, Webster K, Socinski MA. Measuring the side effects of taxane therapy in oncology. Cancer 2003;98:822-31

17. Smith LH. Early clinical detection of ovarian cancer: A review of the evidence. Expert Rev Anticancer Ther 2006;6(7):1045-52.

18. McGuire III WP, Markman M. Primary ovarian cancer chemotherapy: Current standards of care. Br J Cancer 2003;89(Suppl 3):S3-8. 